\title{
Interaction of a quantum-dot cavity system with acoustic phonons: Stronger light-matter coupling can reduce the visibility of strong coupling effects
}

\author{
M. Glässl, ${ }^{1, *}$ L. Sörgel, ${ }^{1}$ A. Vagov, ${ }^{1}$ M. D. Croitoru,,${ }^{1,2}$ T. Kuhn, ${ }^{3}$ and V. M. Axt ${ }^{1}$ \\ ${ }^{1}$ Institut für Theoretische Physik III, Universität Bayreuth, 95440 Bayreuth, Germany \\ ${ }^{2}$ Laboratoire Onde et Matière d' Aquitaine, Université Bordeaux 1, 33400 Talence, France \\ ${ }^{3}$ Institut für Festkörpertheorie, Universität Münster, 48149 Münster, Germany
}

(Received 23 January 2012; revised manuscript received 11 May 2012; published 20 July 2012)

\begin{abstract}
We present a numerically complete study of the combined dynamics of a quantum dot exciton coupled to a single quantized cavity mode and a continuum of acoustic phonons. We demonstrate that acoustic phonons have a pronounced impact on effects characteristic of the strong light-matter coupling regime, such as vacuum Rabi oscillations and collapse and revival scenarios. This impact is considerable already at zero temperature, where initially no phonons are present. Counterintuitively it is found that an increase of the light-matter coupling does not necessarily enhance the visibility of strong-coupling effects. In fact, for typical experimental situations, a stronger light-matter coupling will considerably reduce the visibility.
\end{abstract}

DOI: 10.1103/PhysRevB.86.035319 PACS number(s): 78.67.Hc, 42.50.Md, 42.50.Pq, 78.20.hb

\section{INTRODUCTION}

Reaching the regime of strong light-matter interaction has revealed a rich phenomenology of fascinating physics. A key experiment to provide evidence for the strong-coupling regime is the observation of vacuum Rabi oscillations (ROs), which have been demonstrated in atomic ${ }^{1}$ as well as in solid-state physics. ${ }^{2-4}$ More recently, even a transient switching of vacuum ROs has been shown. ${ }^{5}$ Further intriguing examples for strong-coupling effects are the collapse and revival phenomenon which was first observed in experiments on one-atom maser systems, ${ }^{6}$ the effect of photon antibunching in high finesse microcavities, ${ }^{7}$ and the on-demand generation of entangled photons in a cascaded biexciton-exciton decay in a quantum dot (QD) cavity system. ${ }^{8}$ The latter two are of crucial importance for applications in quantum cryptography ${ }^{9}$ and quantum computation ${ }^{10}$ as well as for quantum optical tests of fundamental aspects of quantum mechanics. ${ }^{11}$

In QD based systems the interaction of carriers with phonons is of utmost importance and represents currently a highly active focus of research. Already for laser-driven QDs without a cavity the phonon coupling leads to various remarkable dynamical features such as a nonmonotonic temperature dependence of the initial decay, ${ }^{12}$ an undamping of ROs at high pulse areas, ${ }^{13,14}$ and a crossover between two qualitatively different relaxation scenarios in an exciton-biexciton system. ${ }^{15}$ Recent studies concerning the impact of acoustic phonons on QD cavity dynamics have revealed a surprisingly strong QD cavity coupling when the cavity is detuned from the QD resonance ${ }^{16,17}$ and a pronounced spectral broadening of the Mollow sidebands through off-resonant cavity emission. ${ }^{18,19}$ Furthermore, it has been predicted that the coupling of longitudinal optical phonons to a QD cavity system may induce a change from classical to nonclassical photon statistics. ${ }^{20}$

In this paper we analyze the combined dynamics of a single cavity mode coupled to a QD interacting with a continuum of longitudinal acoustic (LA) phonons. The light-matter coupling is taken to be in the strong-coupling regime. A special focus is put on studying collapse and revival phenomena well known from the Jaynes Cummings (JC) model. ${ }^{21,22}$ We demonstrate that even at zero temperature LA phonons significantly modify the collapse and revival scenarios. At higher temperatures, the revivals are eventually fully suppressed. In contrast to common intuition we find that for parameters commonly accessible in experiments a stronger light-matter coupling, although it pushes the system further into the strong-coupling regime, reduces the visibility of typical strong-coupling effects. This remarkable finding can be traced back to the resonance character of the carrier-phonon interaction.

\section{MODEL}

Recently, we have presented a real-time path-integral approach that allows for a numerically complete treatment of a LA phonon coupled QD driven by a classical light field. ${ }^{23}$ For the present studies we extended our implementation to the case where the classical field is replaced by a single quantized cavity mode. This gives us the unique opportunity to perform calculations of the phonon-coupled QD cavity system without further approximations, which seems not to have been realized before. Considering the QD as an electronic two-level system, our model Hamiltonian reads

$$
\begin{aligned}
H= & \hbar \omega_{X}|X\rangle\langle X|+\hbar \omega_{C} a^{\dagger} a+\hbar g\left(a^{\dagger} \sigma_{-}+a \sigma_{+}\right) \\
& +\sum_{\mathbf{q}} \hbar \omega_{\mathbf{q}} b_{\mathbf{q}}^{\dagger} b_{\mathbf{q}}+\sum_{\mathbf{q}} \hbar\left(g_{\mathbf{q}} b_{\mathbf{q}}+g_{\mathbf{q}}^{*} b_{\mathbf{q}}^{\dagger}\right)|X\rangle\langle X| .
\end{aligned}
$$

We take the energy of the electronic ground state $|G\rangle$ to be zero, denote the energy gap between $|G\rangle$ and the excited singleexciton state $|X\rangle$ by $\hbar \omega_{X}$, and define $\sigma_{+}=|X\rangle\langle G|, \sigma_{-}=$ $|G\rangle\langle X| . a^{\dagger}(a)$ is the cavity mode creation (annihilation) operator, $\omega_{C}$ is the mode frequency, and $g$ is the QD cavity coupling strength. Here, we concentrate on a resonant QD cavity system assuming that $\omega_{C}$ is in resonance with the polaron-shifted exciton transition. The operator $b_{\mathbf{q}}^{\dagger}\left(b_{\mathbf{q}}\right)$ creates (destroys) a LA bulk phonon with wave vector $\mathbf{q}$ and energy $\hbar \omega_{\mathbf{q}}=\hbar c_{s} q$, with $c_{s}$ being the longitudinal sound velocity. $g_{\mathbf{q}}=g_{\mathbf{q}}^{\mathrm{e}}-g_{\mathbf{q}}^{\mathrm{h}}$ denotes the exciton-phonon coupling constants, where $g_{\mathbf{q}}^{\mathrm{e}(\mathrm{h})}$ are the deformation potential couplings of electrons (holes) in the dot. These model assumptions are realistic, e.g., for GaAs-type QDs. ${ }^{12}$ Material parameters are taken from Ref. 24. 

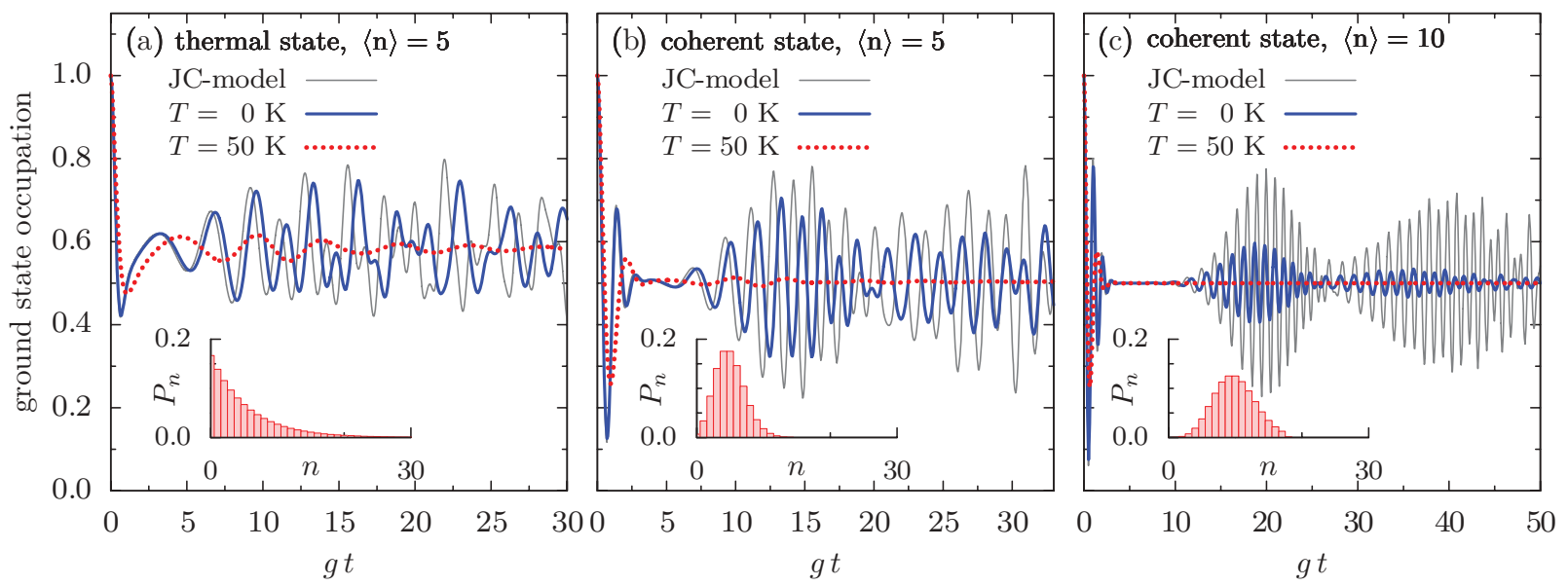

FIG. 1. (Color online) Ground-state occupation $\rho_{00}$ as a function of time for a QD cavity coupling of $g=0.1 \mathrm{ps}^{-1}(\hbar g=65 \mu \mathrm{eV})$ at temperatures of $T=0$ and $50 \mathrm{~K}$ assuming that at $t=0$ the cavity mode was prepared in (a) a thermal state with a mean photon number of $\langle n\rangle=5$, (b) a coherent state with $\langle n\rangle=5$, or (c) a coherent state with $\langle n\rangle=10$. Results without phonon interaction (JC model) are shown as thin gray lines. Insets: initial probabilities $P_{n}$ of the photon number states $|n\rangle$ for the chosen cavity preparations.

\section{RESULTS}

In the following we shall first demonstrate that the coupling to a continuum of acoustic phonons has a significant impact on the electronic dynamics. Shown in Fig. 1 is the time evolution of the ground-state occupation $\rho_{00}$ for a QD cavity coupling of $g=0.1 \mathrm{ps}^{-1}(\hbar g=65 \mu \mathrm{eV})$, which is a typical value in experiments. ${ }^{2,4}$ We assume that at $t=0$ the QD is unexcited, the phonons are in a thermal equilibrium at temperature $T$, and the cavity mode is prepared in a thermal ${ }^{31}$ [Fig. 1(a)] or coherent [Figs. 1(b) and 1(c)] state with a mean photon number of $\langle n\rangle=5$ [Figs. 1(a) and 1(b)] or 10 [Fig. 1(c)], respectively. The corresponding initial probabilities $P_{n}$ for the photon number states $|n\rangle$ are displayed as insets. To provide a reference for the discussion of the phonon influence, we first neglect the exciton-phonon coupling reducing our Hamiltonian to the JC model. ${ }^{21}$ Corresponding results are shown as thin gray lines. For a photon field initially prepared in a coherent state [Figs. 1(b) and 1(c)], we recover the collapse and revival phenomenon. As discussed in the literature, the number of well-resolved revivals strongly depends on the mean photon number. ${ }^{22,25}$ While for $\langle n\rangle=5$ the second collapse is hardly seen [Fig. 1(b)], further collapse and revival sequences become visible for higher mean photon numbers as exemplarily shown in Fig. 1(c) for $\langle n\rangle=10$. Typical cavity experiments are currently working in the low photon number regime. ${ }^{2}$ Therefore, we shall mainly concentrate on $\langle n\rangle=5$ in the following.

Next, we include the exciton-phonon interaction. Interestingly, already at zero temperature (blue solid lines), where initially no phonons are present, the coupling to acoustic phonons considerably affects the dynamics. For both initial cavity preparations we clearly observe a phonon-induced frequency renormalization and a damping compared to the phonon-free case, similar as previously reported in connection with laser-driven QDs without cavity coupling. ${ }^{13,14,26-28}$ Already at $T=50 \mathrm{~K}$ (red dotted lines) the dynamics is completely changed compared to the JC model; e.g., for a cavity mode initially prepared in a coherent state with $\langle n\rangle=5$
[Fig. 1(b)] at $50 \mathrm{~K}$ the dynamics is basically disrupted after the first oscillations and no revival can be seen. For zero temperature, the first revival is clearly visible, but as in the phonon-free case a second well-resolved collapse and revival sequence is absent. The phonon effect on later revivals that become visible for higher mean photon numbers as shown in Fig. 1(c) is similar to that shown in Fig. 1(b) for the first revival, with the tendency that the later further revivals occur the more strongly they are damped. As the damping significantly depends on temperature, the number of well-resolved revivals increases with lower temperature.

Comparing thermal and coherent photon statistics the phonon impact is found to be similar, except for one difference: when the cavity mode is initially in a coherent state [Fig. 1(b)], then at high $T$ and at long times $\rho_{00}$ is close to 0.5 , which is the stationary value for a QD coupled to a classical field, ${ }^{24}$ while it is close to 0.6 when the initial preparation is a thermal state [Fig. 1(a)]. The reason for this finding, that for different initial states the system does not relax to the same stationary state, is the quantum nature of the light field: for a cavity prepared in a Fock state $|n\rangle, \rho_{00}$ performs for all $n \geqslant 1$ phonon-damped ROs until a stationary value of 0.5 is reached. Only for $n=0$ there is no dynamics, as starting in the electronic ground state the system is without excitation and $\rho_{00}(t)=1$ for all times. For a coherent photon state with $\langle n\rangle=5$, the contribution of the Fock state $|0\rangle$ is marginal [cf. inset of Fig. 1(b)], but for a thermal state $|0\rangle$ is the most probable Fock state [cf. inset of Fig. 1(a)], it considerably contributes and shifts the final occupation to a value of $P_{0}+\frac{1}{2}\left(1-P_{0}\right)$.

Most remarkable is the dependence of the dynamics on the QD cavity coupling $g$ as shown in Fig. 2 for a cavity prepared in a coherent state with $\langle n\rangle=5$ at $T=10 \mathrm{~K}$. For $g=0.1 \mathrm{ps}^{-1}$ (red solid line), we clearly see a revival. Interestingly, pushing the system further into the strongcoupling regime by increasing $g$ from 0.1 to $0.5 \mathrm{ps}^{-1}$ (blue dashed line) significantly reduces the visibility of the revival. This finding is in sharp contrast to the common expectation, that a stronger light-matter coupling enhances coherent effects like the collapse and revival phenomenon known to appear 


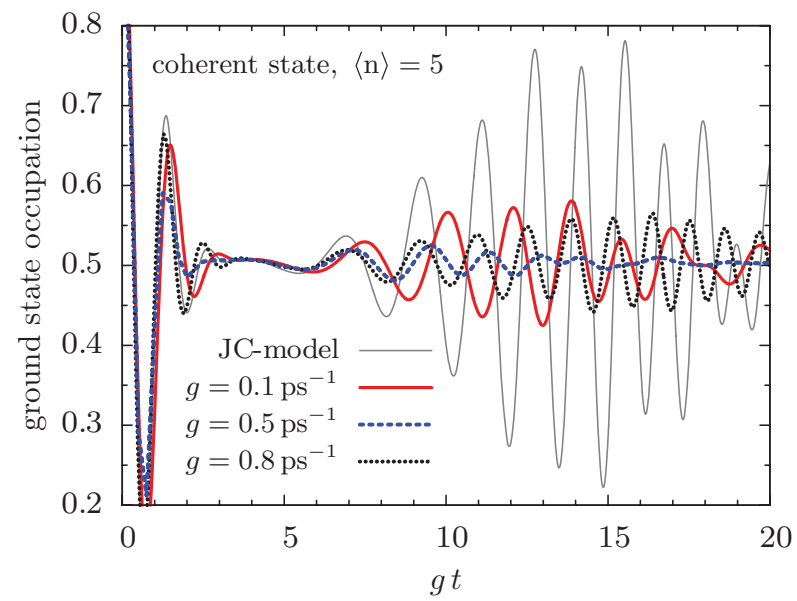

FIG. 2. (Color online) Ground-state occupation $\rho_{00}$ as a function of time for different QD cavity couplings (see key) at $T=10 \mathrm{~K}$ assuming that at $t=0$ the cavity mode was prepared in a coherent state with a mean photon number of $\langle n\rangle=5$. Dynamics without phonon interaction (JC model) are shown as thin gray lines.

only in the strong-coupling regime. ${ }^{22}$ Instead, increasing $g$ to not too high QD cavity couplings leads to a more and more suppressed revival. Clearly visible revivals return only at very strong couplings (e.g., $g=0.8 \mathrm{ps}^{-1}$, black dotted line) that are hard to access in experiments.

To understand the dependence of the damping on the QD cavity coupling $g$ as shown in Fig. 2, it is instructive to analyze first the case of a QD coupled to a cavity mode prepared in a single Fock state $|n\rangle$. In this case $\rho_{00}$ performs damped ROs with a frequency $\omega$ that is renormalized compared to the phonon-free Rabi frequency $f=2 g \sqrt{n}$ as shown exemplarily in the inset of Fig. 3(b) for $n=1, g=0.5 \mathrm{ps}^{-1}$, and $T=$ $10 \mathrm{~K}$. It turns out that the time evolution of $\rho_{00}$ can be well fitted by the expression $\left[1+\cos (\omega t) \exp \left(-\gamma_{d} t\right)\right] / 2$ and that both the damping rate $\gamma_{d}$ as well as the renormalized frequency $\omega$ depend on the QD cavity coupling $g$ and the photon number $n$ solely via the effective coupling strength $g \sqrt{n}$. This allows us to extract $\gamma_{d}$ and $\omega$ as functions of the phonon-free Rabi frequency $f$ by fits to the numerical data. Figure 3 shows corresponding results at $T=10 \mathrm{~K}$. The damping rate [Fig. 3(a)] depends nonmonotonically on $f$ : for small $f$ the damping increases, while for large $f$ it decreases, eventually approaching zero. This behavior is known from a QD coupled to a classical laser field and can be understood by a resonance argument: $:^{13,14,29,30}$ the damping is most pronounced when the Rabi frequency is close to the frequency of the most strongly coupled phonons. Besides the damping, also the frequency renormalization [Fig. 3(b)] is nonmonotonic. For weak light-matter couplings we find that $\omega / f<1$ and that $\omega / f$ increases with rising $g$, while the situation is reversed in the limit of very strong couplings, where the renormalized Rabi frequency approaches its phonon-free value.

It is an important point to note, that the rather involved dynamics in Fig. 2 can be reconstructed using only the curves plotted in Fig. 3: for an arbitrary initial cavity preparation, the Hamiltonian given in Eq. (1) can be decomposed into uncoupled subspaces with different total numbers of excitonic

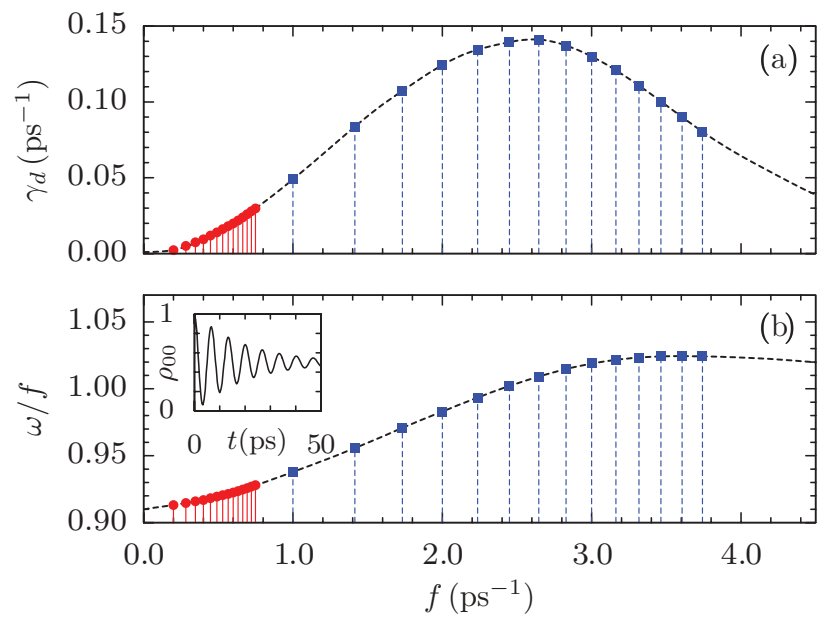

FIG. 3. (Color online) (a) Damping rate $\gamma_{d}$ and (b) frequency renormalization $\omega / f$ of Rabi oscillations for a QD coupled to a cavity mode prepared in a Fock state $|n\rangle$ as functions of the phonon-free Rabi frequency $f=2 g \sqrt{n}$ at $T=10 \mathrm{~K}$. Vertical lines indicate the decomposition of a coherent photon state with $\langle n\rangle=5$ into Fock states $|n\rangle$ with $n=1, \ldots, 14$ for $g=0.1$ (red solid lines) and $0.5 \mathrm{ps}^{-1}$ (blue dashed lines). Inset of (b): $\rho_{00}(t)$ for $n=1, g=0.5 \mathrm{ps}^{-1}$, and $T=10 \mathrm{~K}$.

and photonic excitations. Within each subspace (for $n \neq 0$ ) the phonon-coupled exciton-photon system performs damped ROs between the states $|G, n\rangle$ and $|X, n-1\rangle$, where the damping and the frequency can be taken from Fig. 3. $\rho_{00}$ is obtained by summing over all contributing subspaces, whereby the weighting factor $P_{n}$ of each term is given by the decomposition of the initial photon state into Fock states. ${ }^{32}$ The corresponding values of $\gamma_{d}$ and $\omega$ that are needed to reconstruct the result for $g=0.1 \mathrm{ps}^{-1}$ plotted in Fig. 2 are marked by solid lines and points in Fig. 3. When $g$ is increased to $g=0.5 \mathrm{ps}^{-1}$, the vertical lines shift to higher values (displayed as dashed lines). Note that in this case the involved damping rates are much larger and that the most probable Fock states correspond to damping rates close to the maximal one. This leads to a drastic deterioration of the visibility of the revival. For $g=0.8 \mathrm{ps}^{-1}$ the vertical lines in Fig. 3 are shifted to even higher values (not shown), the most probable states correspond to weaker dampings, and thus the revival is better seen compared to $g=0.5 \mathrm{ps}^{-1}$.

The dependencies shown in Fig. 3 are also reflected in interesting spectral features. Plotted in Fig. 4(a) is the absolute value of the real part of the spectral representation of the polarization, $\rho_{01}(\omega)$, for a QD coupled to a cavity prepared in a Fock state for different values of the phonon-free Rabi frequency $f=2 g \sqrt{n}$ at $T=10$ (dotted lines) and $50 \mathrm{~K}$ (solid lines). Each spectrum consists of a central line and two phononbroadened side-peaks separated from the central line by the phonon renormalized Rabi frequency. At higher $T$, the sidepeaks become not only wider and lower but are also noticeably shifted, reflecting that the frequency renormalization shown in Fig. 2(b) strongly depends on $T$. For small or moderate values of the light-matter coupling $g$ an increase of $g$ (and thereby of $f$ ) at a fixed temperature considerably broadens the side-peaks and lowers their maximal height. Figure 4(b) shows the full width at half maximum (FWHM) of the side-peaks as 

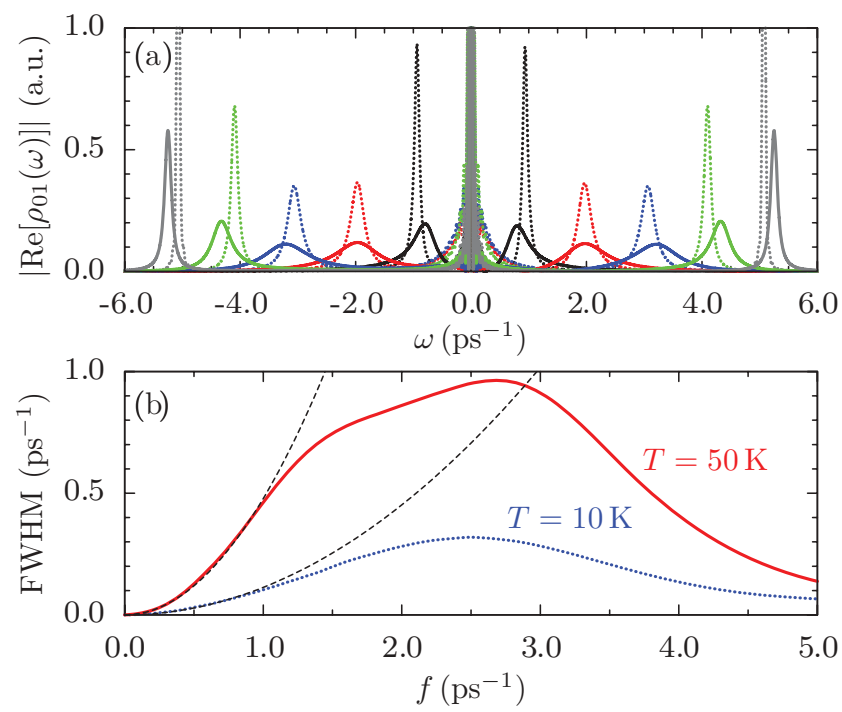

FIG. 4. (Color online) (a) $\left|\operatorname{Re}\left[\rho_{01}(\omega)\right]\right|$ for a QD coupled to a cavity mode prepared in a Fock state with $f=1.0$ (black lines), 2.0 (red lines), 3.0 (blue lines), 4.0 (green lines), and $5.0 \mathrm{ps}^{-1}$ (gray lines) at $T=10$ (dotted lines) and $50 \mathrm{~K}$ (solid lines). (b) FWHM of the side-peaks as a function of the phonon-free Rabi frequency $f=2 g \sqrt{n}$ at $T=10$ and $50 \mathrm{~K}$ together with quadratic fits (black dotted lines) in the regime of small $f$.

a function of $f$. In the low-frequency limit, the FWHM rises almost quadratically with $f$ (dotted lines display quadratic fits). This finding is in line with recent experimental and theoretical works reporting on a quadratic dependence of the FWHM of the Mollow sidebands in fluorescence spectra on the strength of the cw pump. ${ }^{18,19}$ However, at high $f$ a quadratic dependence does no longer persist, as the overall dependence is nonmonotonic, reflecting again the resonance character of the carrier-phonon coupling. ${ }^{13,29}$

\section{CONCLUSIONS}

In summary, we have performed full real-time path-integral simulations of the dynamics of a QD in a cavity coupled to acoustic phonons starting from different cavity-photon statistics. The interaction with phonons causes a strong damping as well as a pronounced frequency renormalization of Rabi oscillations even at zero temperature. At elevated temperatures it completely suppresses the revivals that are typical for the phonon-free Jaynes-Cummings model. In the spectral regime Rabi oscillations manifest themselves as sidepeaks separated from the main transition by the renormalized Rabi frequency. The phonon-induced broadening of these side-peaks is found to depend nonmonotonically on the strength of the light-matter coupling, which can be explained by the resonant nature of the QD-phonon coupling. In the regime of small Rabi frequencies, which is tested in typical experiments, the broadening increases as $g^{2}$, while for very high Rabi frequencies it eventually decreases. The width of the side-peaks is directly related to the visibility of Rabi oscillations or Jaynes-Cummings revivals in the time domain. Indeed, in clear contrast to widespread expectations, for parameters commonly accessed in experiments the visibility of strong-coupling effects is reduced when the light-matter coupling is increased. We believe that our results have far reaching implications on the development and design of future applications of cavity-based devices, as for such applications one aims to maximize typical strong-coupling effects which according to our analysis cannot be achieved by simply trying to maximize the light-matter coupling strength.

\section{ACKNOWLEDGMENTS}

M.G. gratefully acknowledges financial support from the Studienstiftung des Deutschen Volkes. M.D.C. is supported by the Alexander von Humboldt Foundation as well as by a Marie Curie Intra-European Fellowship Action (Grant No. PIEF-GA-2009-235486-ScQSR). *martin.glaessl@uni-bayreuth.de.

${ }^{1}$ M. Brune, F. Schmidt-Kaler, A. Maali, J. Dreyer, E. Hagley, J. M. Raimond, and S. Haroche, Phys. Rev. Lett. 76, 1800 (1996).

${ }^{2}$ J. P. Reithmaier, G. Sek, A. Löffler, C. Hofmann, S. Kuhn, S. Reitzenstein, L. V. Keldysh, V. D. Kulakovskii, T. L. Reinecke, and A. Forchel, Nature (London) 432, 197 (2004).

${ }^{3}$ G. Khitrova, H. M. Gibbs, M. Kira, S. W. Koch, and A. Scherer, Nature Physics 2, 81 (2006).

${ }^{4}$ K. Hennessy, A. Badolato, M. Winger, D. Gerace, M. Atatüre, S. Gulde, S. Fält, E. L. Hu, and A. Imamolu, Nature (London) 445, 896 (2007).

${ }^{5}$ G. Günter et al., Nature (London) 458, 178 (2009).

${ }^{6}$ G. Rempe, H. Walther, and N. Klein, Phys. Rev. Lett. 58, 353 (1987).

${ }^{7}$ D. Press, S. Götzinger, S. Reitzenstein, C. Hofmann, A. Löffler, M. Kamp, A. Forchel, and Y. Yamamoto, Phys. Rev. Lett. 98, 117402 (2007).

${ }^{8}$ R. Stevenson, R. J. Young, P. Atkinson, K. Cooper, D. A. Ritchie, and A. J. Shields, Nature (London) 439, 179 (2006).
${ }^{9}$ N. Gisin, G. Ribordy, W. Tittel, and H. Zbinden, RMP 74, 145 (2000).

${ }^{10} \mathrm{M}$. A. Nielsen and I. Chuang, Quantum Computation and Quantum Information (Cambridge University Press, Cambridge, 2000).

${ }^{11} \mathrm{~S}$. Haroche and J. Raimond, Exploring the Quantum, 1st ed. (Oxford University Press, Oxford, 2006).

${ }^{12}$ A. Vagov, V. M. Axt, T. Kuhn, W. Langbein, P. Borri, and U. Woggon, Phys. Rev. B 70, 201305(R) (2004).

${ }^{13}$ A. Vagov, M. D. Croitoru, V. M. Axt, T. Kuhn, and F. M. Peeters, Phys. Rev. Lett. 98, 227403 (2007).

${ }^{14}$ M. Glässl, M. D. Croitoru, A. Vagov, V. M. Axt, and T. Kuhn, Phys. Rev. B 84, 125304 (2011).

${ }^{15}$ M. Glässl, M. D. Croitoru, A. Vagov, V. M. Axt, and T. Kuhn, Phys. Rev. B 85, 195306 (2012).

${ }^{16}$ S. Ates, S. M. Ulrich, A. Ulhaq, S. Reitzenstein, A. Löffler, S. Höfling, A. Forchel, and P. Michler, Nature Photonics 3, 724 (2009).

${ }^{17}$ P. Kaer, T. R. Nielsen, P. Lodahl, A. P. Jauho, and J. Mørk, Phys. Rev. Lett. 104, 157401 (2010). 
${ }^{18}$ C. Roy and S. Hughes, Phys. Rev. Lett. 106, 247403 (2011).

${ }^{19}$ S. M. Ulrich, S. Ates, S. Reitzenstein, A. Löffler, A. Forchel, and P. Michler, Phys. Rev. Lett. 106, 247402 (2011).

${ }^{20}$ A. Carmele, M. Richter, W. W. Chow, and A. Knorr, Phys. Rev. Lett. 104, 156801 (2010).

${ }^{21}$ E. T. Jaynes and F. W. Cummings, Proc. IEEE 51, 89 (1963).

${ }^{22}$ C. C. Gerry and P. L. Knight, Introductory Quantum Optics, 1 st ed. (Cambridge University Press, Cambridge, 2005).

${ }^{23}$ A. Vagov, M. D. Croitoru, M. Glässl, V. M. Axt, and T. Kuhn, Phys. Rev. B 83, 094303 (2011).

${ }^{24}$ M. Glässl, A. Vagov, S. Lüker, D. E. Reiter, M. D. Croitoru, P. Machnikowski, V. M. Axt, and T. Kuhn, Phys. Rev. B 84, 195311 (2011).

${ }^{25}$ N. B. Narozhny, J. J. Sanchez-Mondragon, and J. H. Eberly, Phys. Rev. A 23, 236 (1981).
${ }^{26}$ J. Förstner, C. Weber, J. Danckwerts, and A. Knorr, Phys. Rev. Lett. 91, 127401 (2003).

${ }^{27}$ A. Krügel, V. M. Axt, T. Kuhn, P. Machnikowski, and A. Vagov, Appl. Phys. B 81, 897 (2005).

${ }^{28}$ A. J. Ramsay, T. M. Godden, S. J. Boyle, E. M. Gauger, A. Nazir, B. W. Lovett, A. M. Fox, and M. S. Skolnick, Phys. Rev. Lett. 105, 177402 (2010).

${ }^{29}$ P. Machnikowski and L. Jacak, Phys. Rev. B 69, 193302 (2004).

${ }^{30}$ A. Nazir, Phys. Rev. B 78, 153309 (2008).

${ }^{31}$ Thermal states with high mean photon numbers $\geqslant 1$ can be prepared using intense tunable narrow-band thermal sources, as discussed in Ref. 20.

${ }^{32}$ For a coherent state with $\langle n\rangle=5$, it is sufficient to concentrate on Fock states $|n\rangle$ with $n \leqslant 14$ as $\sum_{n=0}^{14} P_{n}>0.999$; i.e., photon number states with $n>14$ practically do not contribute. 